\title{
Japón en la geopolítica del siglo XXI
}

Pío García*

\section{RESUMEN}

En 1853, Japón fue forzado a salir de su aislamiento feudal y a entroncarse en la red comercial mundial. Su apertura no significó sumisión a los poderes externos, sino la inmersión en la pugna imperialista, de la que saldría derrotado en 1945. Empero, su postración fue pasajera, dado que la nueva contienda de posguerra lo convirtió en aliado incondicional de la misma potencia que con dos bombas atómicas acababa de cercenarle las ambiciones militaristas. El acuerdo estratégico con Estados Unidos se sostuvo incólume aun después de terminada la Guerra Fría. Más aún, la pugna verbal y el despliegue de fuerza en el archipiélago Senkaku/ Diaoyutai, en 2012, facilitó el retorno al poder del ala política más afín al dictado de Washington y a su plan de seguridad en el Pacífico. Hoy en día, el enfrentamiento en el noreste asiático presenta al bloque estadounidensenipo-coreano, por un lado, y al sino-rusonorcoreano, por otro, en una bifurcación que debe ser vista como un nuevo esquema bipolar que garantizará por un tiempo el equilibrio estratégico regional. Es de esperar, sin embargo, progresivas alteraciones en la correlación de fuerzas tanto en esa sección asiática como en el resto del mundo, debido al creciente poder económico, político y militar chino, con impacto sobre los acuerdos geopolíticos vigentes, incluido el nipo-estadounidense.

Palabras clave: Japón, Asia, geopolítica, bipolaridad, integración.

\section{Japan in the 21st Century Geopolitics}

\begin{abstract}
In 1853, Japan was forced to come out of its feudal isolation and become a part of the worldwide business network. Its opening up did not mean surrendering to external powers,
\end{abstract}

* PhD en Filosofía. Profesor de la Facultad de Finanzas, Gobierno y Relaciones Internacionales, Universidad Externado de Colombia, Bogotá (Colombia).pio.garcia@uexternado.edu.co

Recibido: 30 de octubre de 2013 / Modificado: 17 de junio de 2014 / Aceptado: 17 de junio de 2014.

Para citar este artículo

García, P. (2015). Japón en la geopolítica del siglo xxi. OASIs, 21, 111-130. DOI: http://dx.doi.org/10.18601/16577558. n21.06 
but rather becoming immersed in an imperialist struggle, from which it would come out defeated in 1945. Nevertheless, Japan's prostration was temporary, given that in the post-war period it became an unconditional ally of the same super power that had blocked its military aspirations with two atomic bombs. The strategic agreement with the USA remained intact even after the Cold War had ended. Moreover, the verbal struggle and show of force in the Senkaku / Diaoyutai Islands, in 2012, facilitated a return to power which was more akin to the dictates of Washington and its security plan in the Pacific political wing. Today, the confrontation in Northeast Asia presents the Korean-American-Japanese block, on one side, and the Sino-Russian-North Korean, on the other, on a fork which must be seen as a new bipolar scheme which will guarantee the regional strategic equilibrium. However, progressive changes are expected in the balance of power in both the Asian sector and the rest of the world, due to the impact of growing Chinese economic, political and military power on geopolitical agreements, including the Japanese-American one.

Key words: Japan, Asia, geopolitics, bipolarity, integration.

\section{INTRODUCCIÓN}

La derrota en agosto de 1945, sepultó los planes japoneses que desde la renovación Meiji, en 1868, estuvieron dirigidos a regir los asuntos asiáticos. Solicitado por ambos lados de la recién establecida dicotomía mundial, el hecho de haber llegado primero con sus tropas inclinó la balanza a favor de Estados Unidos, y el eximperio del Sol Naciente quedó enganchado en la esfera capitalista desde aún antes de destaparse la Guerra Fría. Su función de punta de lanza de la estrategia antisoviética en el Pacífico fue compensada de manera visible con oportunidades económicas, de modo que la recuperación de la base productiva fue veloz y el acceso a las posiciones de primera línea en la jerarquía comercial, financiera y de desarrollo tecnológico tuvo un salto espectacular. Tras renacer de las cenizas, en menos de dos décadas Japón se convertía en adalid de varios ramos productivos y de la oferta de servicios: en 1964 hospedó los juegos olímpicos, mientras capturaba segmentos considerables del mercado de autos, barcos, televisores, radios y otros implementos de hogar. Asimismo, ese año inauguró el shinkansen o tren-bala, el primer vehículo de esta modalidad en el mundo. En los años setenta, su fortaleza financiera y productiva competía con la estadounidense, con alcances tales que algunos estudiosos vaticinaron el desplazamiento de este último por el súper Estado asiático (Kahn, 1970; Vogel, 1980). Dicho relevo, en efecto, nunca sucedió.

Dos barreras insuperables se interpusieron en los planes de algunos líderes nacionalistas japoneses que sí consideraban viable poner al país en el centro de las decisiones planetarias. En primer lugar, el Gobierno entró en una fase de crecientes limitaciones presupuestales. Tras una recuperación rápida de los choques petroleros de los años setenta, advino el estancamiento industrial progresivo que obligó al Gobierno a incrementar la deuda en forma sostenida, hasta llegar a representar el 228\% del PIB, en 2013 (oECD, 2013). De hecho, el 
aparato productivo resultó asfixiado por la escasez de trabajadores jóvenes y la estrechez del mercado interno, insuficientes ambos para atender las exigencias de una economía globalizada. Las obligaciones ante las demandas de sus socios ricos lo obligaron, asimismo, a aceptar medidas que aceleraron aún más la contracción productiva; de manera más concreta, la determinación del G-5 ${ }^{1}$ de revaluar el yen, en 1985, le eliminó de inmediato el superávit comercial. En segundo lugar, la dependencia política y estratégica de Estados Unidos dio paso a una relación descompensada que impidió la internacionalización más armónica de la sociedad japonesa. De hecho, durante setenta años el país no tuvo una opción distinta a la diplomacia económica para vincularse a la comunidad mundial, porque el resto fue diseńado en Washington, a partir del Tratado de Seguridad, suscrito en San Francisco, en 1951. En adelante, la alianza entre los dos significó la unidad de sus intereses políticos y de participación arreglada en los organismos multilaterales. Este fue el patrón propio de la política exterior japonesa en la segunda mitad del siglo $x x$.

En las dos primeras décadas del siglo XXI, el sistema global sufre alteraciones sustanciales, con efectos directos sobre el horizonte de acción mundial de Estados Unidos, de Japón y de su tratado de seguridad mutua. Ante todo, y a medida que China robustece su capacidad económica, sus vínculos regionales y globales toman una naturaleza distinta a la que tuvieron en el pasado. Desde un punto de vista de los intereses industriales es comprensible su sensibilidad respecto a las obstrucciones que pueda encontrar para acceder a los bienes requeridos por su aparato productivo, así como para ubicar sus mercancías en los mercados externos. La garantía de esos movimientos depende de equipos y fuerzas militares en las zonas críticas, como pueden ser los mares adyacentes o las vías terrestres asiáticas. A partir de estas renovadas necesidades de protección, la tensión con los esquemas de seguridad de otras potencias en la región, como lo pueden ser Rusia o Estados Unidos, es inevitable.

Cuando China se encamina a superar el tamańo económico estadounidense surgen ciertas preguntas sobre sus efectos políticos y estratégicos: ¿cómo se proyecta hacia su zona contigua y el resto del mundo para defender sus intereses nacionales? ¿Cómo se aprecia en Japón y en Estados Unidos el resurgimiento chino y su desafío como gran potencia? ¿Qué impacto puede seguir recibiendo la alianza militar nippo-estadounidense del liderazgo chino regional y global? En este contexto de variación en el peso de los superpoderes contemporáneos, ¿qué opciones geopolíticas tendrá Japón en las próximas décadas?

Sobre el particular es notable la coincidencia de muchos analistas en forzar sus marcos interpretativos para negar el lugar preponderante que adquiere China en los asuntos mundiales del siglo xxi. Connotados teóricos, como Mearsheimer, minimizan la capacidad del país para enfrentar una estructura de poder muy "estable" debido al dominio que ejercen

1 Compuesto por Estados Unidos, Gran Bretaña, Francia, Alemania e Italia. 
Estados Unidos y sus aliados en el Pacífico occidental. Su perspectiva neorrealista desconoce la capacidad de control geopolítico por parte de las organizaciones multilaterales -lo cual no es desacertado del todo-, dada su supeditación a la competencia entre los grandes poderes. Para él, en el este asiático, como en cualquier otro escenario, priman los intereses estatales, alrededor de los cuales se constituyen las alianzas, los acopios de armamento y diseńos estratégicos y demás sinsabores de la lucha por el poder, en un ambiente de anarquía constante (He, 2013). Pero, según él -volviendo a Mearsheimer, y esto con argumentos menos convincentes- China se proyectaría sobre la zona contigua y hacia el resto del mundo bajo presiones militares estrechando su campo de acción, dado que tales decisiones reforzarían los compromisos de seguridad de Estados Unidos con Japón, Corea, Australia y otros países del Asia y el Pacífico (Mearsheimer, 2001; 2006). Esa interpretación revela un claro sesgo sinófobo. Por su parte, la teoría de los complejos regionales de seguridad considera que la competencia de poder en Asia se ha de explicar como un sistema con cierta independencia de la pugna por el poder global, ya que por ahora ningún Estado puede superar la hegemonía estadounidense (Buzan y Wæver , 2003), explicación que se obliga a empequeńecer el rol chino por el simple deseo de magnificar el control mundial americano. Otro prejuicio antichino fácil de advertir. A su vez, hay quienes van más allá y en una actitud de conmiseración insisten en poner el vigor de la sociedad china al servicio de Washington en su choque interminable con Rusia, su competidor atómico (Brzezinski, 1998). Como he- mos de explicar, en este tipo de interpretación las capacidades de influencia de China en los asuntos planetarios son recortadas, ante todo por las prevenciones que soportan una visión global centralizada en el papel rector político, militar y valorativo estadounidense, en manifiesto contraste con un escenario internacional cada vez más poshegemónico.

En contra de los análisis prejuiciados, nuestro argumento afirma que, a pesar de la voluntad firme de los Gobiernos de Japón y Estados Unidos de preservar su alianza estratégica sobre la base de la cooperación militar comprendida en el Tratado de Seguridad, la transformación del sustrato económico y militar que eleva el rango internacional chino le crea presiones estratégicas a Tokio, pero al mismo tiempo le tiende lazos favorables para su relacionamiento regional. En efecto, en cuanto logre resolver los problemas frente a la afiliación global japonesa y las modalidades de cooperación en el ámbito asiático estimulen la discusión política, sus Gobiernos podrán acoger redefiniciones partidistas y renovar su modelo productivo a favor de los vínculos colaborativos con el vecindario. El plan de exposición comprende la revisión de la alianza militar con Estados Unidos, el análisis del nuevo escenario asiático y mundial, y las consideraciones sobre sus efectos directos en los aspectos de la seguridad y la participación internacional de Japón en el futuro cercano.

\section{LA DEPENDENCIA ESTRATÉGICA JAPONESA}

El pueblo japonés ha brindado suficiente soporte a la dependencia militar del país, porque 
es consciente de sus beneficios económicos, aun cuando ha sido una opinión pública desafecta a incrementar el gasto de defensa porque le podría significar algún retroceso en el bienestar alcanzado (Kihl, 1986, 131). Sin embargo, ese mismo pragmatismo puede llegar a ser lesivo para esa comunidad de intereses estratégicos con Estados Unidos. Analicemos, al respecto, el desarrollo de esa alianza, sus logros y las adaptaciones a los nuevos retos domésticos y externos.

Tras su rendición, Japón fue ocupado de inmediato por las tropas del general Douglas MacArthur, comandante supremo de los poderes aliados. El mes de agosto de 1945 fue el más trágico para una sociedad que en una semana sufrió la destrucción total de dos ciudades - con 350.000 víctimas a causa del fuego atómico-y la pérdida de la soberanía, mientras aún se aferraba el delirio de ser el Estado más poderoso de la región y el director y representante asiático ante el resto del mundo. Una vez descargadas las bombas sobre Hiroshima y Nagasaki, en los demás países cayó en un silencio cáustico, que encubrió los movimientos tectónicos entre los vencedores, cuando se alumbraba la Guerra Fría. Allí quedó signado el destino japonés como pivote de las posteriores operaciones militares en Corea y Vietnam. La firma del tratado de seguridad en septiembre de 1951 indicó no un comienzo sino el fin de una etapa, a saber: la colaboración militar inicial entre Estados Unidos y Japón, que se remonta a 1947, cuando los planes económi- cos y políticos previstos para democratizar al país hostil fueron desviados con el fin de darle paso a los requerimientos geopolíticos. En efecto, las reformas iniciales diseńadas para resolver las necesidades primordiales de la población omitían la más mínima dotación de fuerza policial y militar, como represalia por la aventura expansionista. Tales medidas de desmilitarización no prosperaron, dado que muy pronto el Pentágono se vio abocado a disponer de nuevas funciones para los Estados ocupados en Asia, de tal modo que desde 1947 Corea y Japón dejaron de ser pueblos accesorios para tomar un puesto frontal en el bloqueo al avance comunista (Nakamura, 1988, p. 187). Bajo estas condiciones de bipolaridad estratégica, el factor de las armas fue primordial y la remilitarización japonesa fue inexcusable.

La resistencia blandida por los socialistas y otras corrientes críticas de la dependencia política y militar de Estados Unidos fue disuelta en forma violenta en enero de 1960 con motivo de la ratificación del acuerdo de seguridad $^{2}$. El primer ministro Hayato Ikeda, quien pocos meses después sucedió a Nobosuke Kishi, ató la alianza externa a los objetivos desarrollistas internos, encarrilando el sistema productivo al crecimiento elevado y sostenido de las dos décadas siguientes. Los mecanismos distributivos del "plan para duplicar el ingreso" y las "políticas de paciencia y reconciliación" funcionaron lo suficiente como para poder satisfacer las demandas básicas de la población, cuya aceptación del patronazgo autoritario del

2 La Dieta confirmó el Pacto de Asistencia Mutua en Seguridad, de 1954, y el texto revisado del acuerdo de defensa denominado Tratado de Seguridad y Cooperación Mutua entre Estados Unidos y Japón. 
Partido Liberal Demócrata de Japón (PLD) se sostuvo hasta el final del ciclo económico expansivo (Packard, 2010). En ese ambiente de prosperidad no hubo posibilidades de mayores descalificaciones a la dependencia de Estados Unidos, vista dicha anormalidad más bien como contraprestación por el servicio prestado en la competencia estratégica orbital; en consecuencia, las manifestaciones de insatisfacción con el Tratado fueron esporádicas.

Con el paso del tiempo, la alianza se hizo más compleja y temeraria. En 1992 fue denunciada y debatida en el parlamento la decisión del Gobierno de participar con las Fuerzas de Autodefensa en el plan de paz para Camboya implementado por la ONU. El punto de la controversia radicó en el envío de las tropas al extranjero, en un claro desvío del artículo 9 de la Constitución Nacional, que prohíbe inmiscuir al país en conflictos armados externos. Hasta entonces, el apoyo a las acciones militares de la ONU se había ajustado al envío de recursos financieros. En la línea tradicional de estos aportes, una contribución abultada de US\$13.000 millones constituyó el apoyo a la guerra contra Iraq para sacar las tropas de Sadam Hussein de Kuwait, en 1991. La luz verde para el envío de tropas a Camboya alentó el acompañamiento de las operaciones bélicas estadounidenses aún por fuera del dominio del Consejo de Seguridad. De esta manera, las Fuerzas de Autodefensa se hicieron presentes en las guerras tempranas del presente siglo en Afganistán e Irak (Stengel, 2008).
El Tratado le implicó a Japón ceder su autonomía militar, disponer de recursos para sufragar los costos del personal estadounidense en el país y albergar a esa fuerza a lo largo del archipiélago. El mayor contingente se ubicó en Okinawa, territorio administrado por Estados Unidos hasta 1972. Allí ocurrieron los más sonados casos de rechazo popular a los militares extranjeros, por varios motivos, tales como el excesivo espacio ocupado por las tropas, equivalente a la quinta parte de la extensión de la prefectura, en una zona sin tierra para la producción agrícola; la contaminación y otros efectos nocivos de las operaciones diarias en la base, al igual que la conducta delincuencial de algunos soldados involucrados en desmanes de embriaguez y abuso sexual de menores (Eldridge, 2001). Tras seis décadas de denuncia y movilización de los habitantes de Okinawa, su lucha fue más efectiva para lograr cambios en el acuerdo que las críticas de los partidos políticos y movimientos populares en otros lugares del país adversos al abandono de la política pacifista, incluida la abstención de participar en conflictos en el extranjero. De esta manera, en 2006, ambos Gobiernos acordaron trasladar parte de las facilidades militares y del personal de la base de Futenma a Guam³ ${ }^{3}$ la más sureña de las Islas Marianas, posesión de Estados Unidos en el Pacífico Occidental.

La rentabilidad económica de la alianza de seguridad está representada en el crecimiento explosivo del aparato industrial, la captura

3 Incluye la estación aérea del cuerpo de marina con 8 mil soldados (Chanlett-Avery, 2012). 
de los mercados externos y el control de tecnologías de punta por parte de las empresas japonesas. Desde un comienzo, el Ministry of International Trade and Industry (MITI) pudo diseñar planes a largo plazo que condujeron al desarrollo productivo desde la oferta de bienes intensivos en mano de obra hasta los productos intensivos en capital y tecnología. El país logró pasar, así, de la producción de manufacturas livianas, a la industria pesada y luego liderar sectores de la electrónica, la biotecnología, las ciencias del espacio, entre otros (Johnson, 1982; Nakamura, 1988). Es probable que preservar por treinta años el segundo puesto del escalafón de las mayores economías no hubiera sido posible sin el gasto por encima del 3\% del PIB en I\&D, gracias a una inversión en defensa por debajo del $1 \%$. Una buena parte del dominio tecnológico japonés estuvo nutrido por los favores de su regente militar estadounidense, que además auspició la presencia de representantes japoneses en los puestos de mando de los organismos multilaterales como el Fondo Monetario Internacional (FMI), UNEsCo, la Agencia Internacional de Energía Atómica (AIEA) o la Organización Internacional para las Migraciones (OIM). De hecho, en las cuatro décadas que siguieron a la derrota y administración militar por parte de Estados Unidos, el Gobierno japonés cosechó éxitos estruendosos derivados de la cooperación estratégica, a cambio de aceptar el encargo de principal aliado en la región del Pacífico asiático. Otros miembros de la coalición en la región-Corea ${ }^{4}$, Australia, Singapur, entre otros- tuvieron un encargo menor. Estos frutos no tienen, empero, garantizada su continuidad.

La prosperidad japonesa a expensas del cubrimiento militar estadounidense despertó la inquietud en las demás potencias industriales. Con el fin de no revivir los fantasmas de su pasado ultra nacionalista, en vez de pedirle elevar el gasto militar por el encima del $1 \% \mathrm{del}$ PIB, se le intervino la tasa de cambio, según la decisión tomada por el G-5 en la reunión en el Hotel Plaza de Nueva York, en 1985. En ese momento, las economías grandes determinaron revaluar el yen por encima del $100 \%$, con el ánimo de invertir las tendencias del comercio y abrir el mercado del único país asiático industrializado a los productos estadounidenses y de otros socios industriales ${ }^{5}$. El resultado inmediato fue el ensanchamiento adquisitivo japonés cuyas empresas se volcaron a adquirir bienes raíces en Estados Unidos y activos por todo el mundo. Esta racha, por cierto, fue bastante corta, de modo que tras la fugaz etapa de adquisiciones vino una era de parálisis de su aparato industrial, en razón de la pérdida de competitividad de las exportaciones frente a sus vecinos asiáticos principalmente. El receso productivo japonés desde 1990 fue paralelo al

\footnotetext{
4 En un cambio curioso y diciente, en años recientes la ofensiva comercial coreana ha sido estimulada por la relación favorable de sus empresas con los avances tecnológicos en Estados Unidos, en una cooperación que contrajo el poderío japonés en el campo de las TIC (Rhyu, 2006).

El superávit comercial japonés de US\$46 mil millones con Estados Unidos, se convirtió como efecto de tales medidas en déficit de US\$12 mil millones con el resto del mundo en mayo de 2013 (Salem Press, 2009; Trading Economics, 2013).
} 
surgimiento económico de los Tigres Asiáticos y China, fenómeno que las inversiones niponas fomentaron en parte.

En el inicio del siglo XxI encontramos la alianza nippo-estadounidense con dificultades inocultables. Hacia el año 2000, mientras Japón empezaba una nueva década de merma productiva interna, Estados Unidos elevaba su voluntad hegemónica a unos costos militares insospechados, en su afán por desbaratar el "terrorismo". La opción escogida por este último para estimular tanto el frente externo como el interno con el objeto de preservar su posición dominante fue la de abrir nuevos focos de guerra en Afganistán en 2001 y, dos ańos después, en Irak. La estrechez presupuestal japonesa no fue óbice para acompañar a Washington en ambos acometidos billonarios ${ }^{6}$, a pesar del hecho de que participar en una forma tan intensa no es una medida de fácil venta a la opinión pública, dado el freno persistente en el desempeño productivo del país.

Como parte de los cambios diseńados para hacer más amable la colaboración militar, ambos Gobiernos acordaron readecuar las instalaciones en Okinawa y trasladar los marines de Futenma a Guam, con el fin de mermar el personal militar en esa zona, a un costo de US\$26 mil millones para las arcas japonesas (Yoda, 2006). Por esos años, el deterioro productivo terminó por desacreditar el liderazgo de los liberales ${ }^{7}$, quienes salieron del Gobierno en 2009; sin embargo, los nipodemócratas ${ }^{8}$ que los reemplazaron durante los cuatro ańos siguientes no desconocieron en ningún momento el compromiso estratégico con Washington. En medio de la tensa relación con China desencadenada por la compra del archipiélado Senkaku por parte del Gobierno central, el electorado acogió una vez más el discurso nacionalista del PLD y la férrea alianza militar con Estados Unidos, retornándole el mando del país?

\section{LA REORGANIZACIÓN REGIONAL ASIÁTICA Y LA SEGURIDAD JAPONESA}

La fase de la historia asiática iniciada en 1990, cuando la disputa estratégica entre Estados Unidos y la urss llegó a su fin, podría ser identificada como la asianización asiática ${ }^{10}$. Hasta ese momento, el más extenso y poblado de los continentes había sido un lugar de disputa entre esos poderes externos y antes de la Segunda Guerra Mundial como zona de la intensa disputa imperialista. El sometimiento a las potencias extranjeras había empezado con

\footnotetext{
6 Los costos de estas guerras, al terminar 2012, fueron calculados en US\$6 billones (Bilmes, 2013).

7 El equipo gubernamental del Partido Liberal Democrático.

8 Hace referencia a los votantes del Partido Democrático de Japón, presidido en ese entonces por Ichiro Ozawa y el premier Yukio Hatoyama.

9 En febrero de 2013, a pocas semanas en el cargo, Abe ratificó en Washington la alianza de seguridad, cuya "robustez nadie debe poner en duda” (Takenaka, 2013).

10 Concepto acuñado por el periodista Yoshi Funabashi (1993).
} 
la llegada de los portugueses y espańoles en el siglo $\mathrm{xv}$, pero fue exacerbado en el siglo XIX, cuando la ofensiva tuvo por objeto a China y a Japón, los dos imperios renuentes a vincularse al circuito económico global. Un tutelaje similar a la doctrina Monroe, por medio de la cual Estados Unidos reivindicó el derecho de aislar al continente americano de las ansias imperialistas europeas, lo aplicó Japón desde 1895, a los países vecinos por medio del proyecto de la Esfera de Coprosperidad de los Pueblos Asiáticos, ideado por Ikki Kita (García, 2001). La excusa del retiro de los ocupantes europeos de los países asiáticos condujo al apetito colonialista japonés a su propia destrucción en 1945. Entre tanto, las cinco décadas de expansión violenta dejaron una estela de sangre en el oriente de Asia, suficiente como para alimentar un resentimiento que no logra borrar aún. Nada impide que con menos resistencias, sea China el país que enarbole en la primera parte del siglo Xxi la bandera de la autonomía asiática, que buscó alejar la injerencia externa en los asuntos de la región.

Los factores fundamentales del cambio del contexto de la seguridad japonesa y su concomitante efecto sobre la cooperación militar con Estados Unidos tienen que ver con el ascenso chino al rango de gran poder global y su influencia en la integración regional asiática, el sostenimiento de la política de seguridad rusa y el dominio tecnológico civil alcanzado por actores menores pero cercanos como Corea del Sur y Taiwán.
En primer lugar, desde su estatus de gran poder global, la influencia china en los asuntos asiáticos aparece mucho más expresa, continua y determinante. La diplomacia de Beijing capta a favor de su despliegue asiático la ascendencia dentro de los países de la ASEAN, que se remonta al sostén comercial brindado durante la crisis económica de 1997, cuando la recuperación a través del ensanchamiento exportador tuvo en el mercado chino un aliciente básico (García, 2005). El pacto dirigido a crear el extenso mercado de Asia Oriental dio el primer paso con el acuerdo con China, en 2003, seguido después por los pactos similares con Japón y Corea. El área de libre comercio de ASEAN+6 incluye también a Australia, Nueva Zelandia e India, para dar lugar al mayor bloque económico, dentro del cual sobresale el PIB chino por su tamańo y más elevado crecimiento ${ }^{11}$.

La forma como fue concertada la reciente coordinación financiera revela el ámbito ampliado de la cooperación regional. El punto de quiebre de las negociaciones se logró en la Iniciativa Multilateral de Chiang Mai, de 2010, a través de la cual los intentos de aislar el este asiático de los movimientos de monedas fueron atenuados, en procura de la colaboración antes que la rivalidad con el FMI y el Banco Mundial, las instituciones multilaterales más prestantes. Los tres aspectos destacados del proceso fueron la negociación no condicionada de los países fuertes y los de menor desarrollo, la compaginación de los intereses entre las dos economías

11 Por paridad de compra, el PIB chino se calculó en US\$12,1 billones en 2012 y el PIB colectivo de ASEAN en US\$3,7 billones (CIA, 2013). 
mayores de China y Japón, y la apertura del grupo a las instancias mundiales en contravía del enclaustramiento. La forma particular de establecer acuerdos contribuyó al arreglo financiero que permanece poco institucionalizado y ata una serie de balances calibrados de manera cuidadosa para acomodar los diversos intereses intra y extrarregionales (Ciorciari, 2011). Es por esta división entre los particularistas y los universalistas que la integración de Asia Oriental se proyecta más abierta que otros experimentos de integración regional, y despejó el ambiente para el entendimiento entre japoneses y chinos.

Acuerdos progresivos relativos a la cooperación en ciencia y tecnología, integración física, cooperación social, cultural y educativa han de recibir impulsos sucesivos. En cambio, la cooperación política y estratégica, sobre la cual el influjo chino es anticipable como el factor primordial, tendría una marcha más lenta y contestada. Una parte de los recuerdos molestos saldrá a relucir, en concreto, la hostilidad que despertaron los movimientos maoístas en ciertas élites del Sudeste Asiático, en los años cincuenta y sesenta. Asimismo, la intervención militar en Vietnam en represalia por la ocupación de Cambodia, en 1979, se convirtió en pesadilla china de la que supo salir a tiempo. Comparada con la prevención frente a Japón por la dolorosa memoria de la guerra y sus extravíos colonialistas, la influencia de Beijing parece más aceptable. Además, el decálogo político chino atrae más a la dirigencia asiática que el sistema japonés ceñido en las últimas décadas al ideario neoliberal, el uno estable y auténtico y el otro menos original. Aspectos llamativos para las clases políticas asiáticas son el socialismo con características locales -muy rentable en tiempos de encogimiento de las economías liberales-, el centralismo administrativo y el consenso nacional del Partido Comunista, que garantiza la estabilidad del Gobierno, la cada vez más activa política exterior y el soporte a la institucionalidad multilateral, así como su versión de los derechos humanos y el control de la información por motivos de seguridad nacional. Ese modelo de Estado desarrollista, ya experimentado por Japón y los NIC (Newly Industrialiizyng Countries) (Johnson, 1982; Woronoff, 1992) es, sin duda, sugestivo para varios países de la esfera cultural confuciana.

Pero esos países también advierten cómo el crecimiento económico de China viene aparejado del gasto abultado en defensa y el mejoramiento de su armamento. La disuasión nuclear depende de 340 ojivas; respecto al nuevo equipamiento, las fuerzas armadas ya lanzaron su primer portaaviones en Qingdao, y acrecentaron la aviación con las adquisiciones de tecnología aérea rusa. No hay duda de que el Ejército Popular de Liberación es un verdadero poder global (Wortzel, 2013). Teniendo en cuenta el tamaño colosal de su capacidad productiva, agenciar el dominio sobre las zonas marítimas exclusivas y sobre los corredores marítimos y terrestres es un asunto capital para la dirigencia china, cuando su dependencia del comercio internacional se ha vuelto tan aguda. El rápido aumento disuasivo de EPL, con equipo más sofisticado y personal mejor calificado, suscita inquietudes en la región ante la posibilidad de medidas unilaterales como las adelantadas en el mar del Sur de China, donde disputa la soberanía de los islotes Spratly y Paracel con Vietnam, Filipinas, Malasia y Taiwán. Impedir la percepción 
de un trato arrogante y arbitrario va a poner en juego la habilidad diplomática de Beijing.

En segundo lugar, la capacidad militar no nuclear china ha empezado a desplazar la de Rusia, que por cierto le facilitó la inyección tecnológica en los últimos años; sin embargo, en este reordenamiento regional, la colaboración sino-rusa es fundamental. Una vez superadas las incertidumbres possoviéticas, Rusia encara sus intereses nacionales con una buena dosis nacionalismo y giro en sus alianzas. A la dirigencia moscovita le ha quedado en claro que su afiliación europea no es tan promisoria como el intercambio con Asia, desvío que la mueve hacia los mercados de China, India y Japón, entre otros. Al enlazar esa inmensa población desencadena el aprovechamiento de la cuantiosa riqueza siberiana. En cuanto Rusia se reencuentra a Asia, renueva su interés por el Pacífico y por sus territorios orientales. Las instalaciones que tuvieron motivos iniciales de defensa se convierten en puntales de proyectos extractivos de gas, petróleo, madera y otros bienes primarios para el mercado asiático por medio de oleoductos, gasoductos y vías, financiados con capital chino, coreano y japonés (The Siberian Times, 2013). Ser parte del Foro de Cooperación Económica Asia-Pacífico (APEC, por su sigla en inglés) se agrega a la proyección rusa hacia su flanco del este, una membresía obtenida por la gestión japonesa. Es paradójico para los intereses nacionales de Tokio que la retribución por ese favor sea agridulce, dado que de una parte el acceso a los hidrocarburos rusos se despejó, pero en simultáneo el cerrojo sobre los reclamados "Territorios del Norte" aleja cada vez más la posibilidad de recuperarlos. En consecuencia, las solicitudes japonesas de retomar la soberanía sobre las islas Kuriles ${ }^{12}$ están condenadas a chocar con el muro de una Rusia indiferente y envalentonada.

China y Rusia conjugan su proyección concertada a través de los acuerdos bilaterales de claro contenido económico y militar, con la institucionalización de la cooperación regional centroasiática. El mecanismo ideado para esta última es la Organización de Cooperación de Shanghái, de 2001, que ese año formalizó la experiencia de consultas vivida como Conferencia desde 1994. Ambas potencias resuelven sus asuntos fronterizos y las relaciones con los países de Asia Central, en donde la problemática económica, política y estratégica es múltiple. En efecto, a la dispersión de grupos islámicos radicalizados se agrega el tráfico de estupefacientes y el contrabando de bienes. De igual modo, reposan en esa extensa franja reservas fabulosas de minerales, metales, hidrocarburos y tierras para satisfacer la demanda, en especial china. No sin razón, esa parte del mundo es considerada un eje de la competencia estratégica a causa de la simultánea proyección india, iraní, europea y estadounidense sobre ella, de una manera tan intensa que repite el enfrentamiento entre los imperios zarista y británico, el Gran Juego del siglo xix (Brzezinski, 1998), que incita una alianza contestadora de las acciones de la OTAN en Asia (Wishnick, 2009).

12 Las cuatro islas de Etorofu, Kunashiri, Shikotan Habomai (en la denominación japonesa) fueron tomadas por la URSS en 1945, junto con Sajalín y demás territorios capturados por Japón en 1905. 
De esta forma, algunos de los contendores más definidos, por ahora, son Japón, Sur Corea, Taiwán, Australia y Nueva Zelanda por el lado estadounidense, mientras Rusia, China, Norcorea y Birmania, entre otros, se ubican en la línea contestataria.

Por último, un aspecto adicional que tampoco debe ser omitido dado su impacto para la integración asiática y para el acomodo japonés en ella es el adelanto tecnológico logrado por China y los NIC, cuyo efecto sobre los nichos controlados por las sogo shosha se han hecho formidables. Emblemáticas compañías tipo Sony, gloria japonesa en electrónica, compite en el mercado mundial con los gigantes coreanos Samsung o LG, las taiwanesas Hacer y Asus o Huawei, y demás corporaciones chinas, cuando antes sus referencias eran extrarregionales. La integración industrial asiática sigue el patrón iniciado por Japón en los años sesenta, cuando empezó a trasladar a los países vecinos parte de su producción manufacturera por razones de costos y normas ambientales domésticas, después de la triste experiencia en la bahía de Minamata ${ }^{13}$. Hoy día, Corea del Sur y Taiwán repiten la secuencia con el fin de sacarle rentabilidad a la mano de obra de China y los países del Sudeste Asiático. A diferencia del pasado, cuando la integración tuvo un carácter restringido a las operaciones intraindustriales, el nuevo ciclo cuenta con el marco formal de los acuerdos para vincular las economías vecinas grandes a través del proyecto ASEAN $+6^{14}$, que asocia los países del sudeste con China, Japón, Corea, Australia, Nueva Zelandia e India.

\section{NUEVOS RUMBOS GEOPOLÍTICOS JAPONESES}

Al comenzar la segunda década del siglo XxI, el PIB chino remontó al japonés, tras lo cual se enfiló a equiparar y superar el estadounidense. Solo unos pocos analistas anticiparon este despliegue $^{15}$ en los ańos setenta, cuando Nixon y su secretario de Estado, Kissinger, descongelaron las relaciones con Mao y fracturaron el bloque socialista. Desde entonces, gracias a la inyección constante de capital y tecnología externa, el primer contingente laboral del mundo ha sido utilizado para consolidar un centro manufacturero connotado. La solución no pudo ser más gratificante para aquellos inversionistas ávidos de renovada oferta y nichos comerciales expandidos, de modo que la oferta china de manufacturas de bajo costo capturó el mercado global. El problema para

13 La población sufrió el deterioro nervioso a causa del pescado contaminado con el mercurio vertido al mar por la fábrica de químicos Chisso, instalada a comienzos del siglo xx. En varias localidades japoneses se registraron casos similares, que por mucho tiempo el Gobierno central se negó a reconocer.

14 El proyecto de integración subregional y regional sigue priorizando los aspectos económicos sobre los militares (Friedrichs, 2012); sin embargo, la ascendencia china sobre el vecindario va a requerir programas viables de su parte para elevar las medidas de confianza que conduzcan a remover las justificaciones que algunos países alegan para continuar aliados a Estados Unidos.

15 Immanuel Wallerstein (1974), entre esos pocos. 
la alianza regidora del poder mundial es que los dirigentes en Beijing no se dejaron seducir por los cantos de sirena de la apertura frenética de su mercado -como sí lo hicieron los rusos con los resultados catastróficos tan conocidos-, y sostuvieron un Estado fortificado en forma paralela a la expansión económica. Como es fácil entender, la captación sostenida de réditos eleva el gasto de defensa, moderniza el equipamiento, financia las operaciones y las alianzas militares, gracias a lo cual la República Popular coopta a Rusia y su frente común define la contraposición estratégica fundamental, en una forma de bipolaridad restablecida. De esta manera, sus protegidos pueden apuntalar sus posiciones en Siria, Irán o Norcorea.

Por supuesto, no es ese último país con su resistente voluntad nuclear el desafío prevalente del croquis geopolítico de Washington y sus aliados en el Pacífico Occidental. La opinión pública japonesa es muy sensible a la información sobre las amenazas de Pyongyang, las cuales son amplificadas para darle beneficio al gobierno de turno y al robustecimiento militar del otrora poderoso imperio (Hughes, 2009). Pero el tamaño de la República Popular Democrática de Corea es irrisorio comparado con el poder japonés: su PIB es de apenas el $2 \%$ de este, lo que la ubica en el puesto 125 en la escala mundial, hallándose limitada en sus proyecciones militares, como es evidente. Se trata, entonces, de un contendor accesorio, que carece aún de las armas disuasivas. Es por esta razón que habría que ver la permanencia de los acuerdos estadounidenses con Japón y Corea, y las ofertas de apoyo militar a Vietnam, Malasia y Singapur, entre otros países de Asia Oriental, más bien como piezas de un esquema estratégico para cercar a China o prevenir cualquier avance sino-ruso sobre el Pacífico. Aún más, ese control se extiende a los otros flancos del país hacia el sur, a través de la colaboración con India, en ciertas circunstancias, o con Paquistán, en otras, lo mismo que a su frontera occidental, a través de las bases en Kazajistán, Tayikistán y Kirguistán y las fuerzas de la OTAN en Afganistán, todos países fronterizos chinos.

Frente a la ofensiva de los estadounidenses y sus aliados, la alta plana política en Beijing no se ha quedado ni impávida ni resignada. Todo lo contrario: asegurar su puesto de mando en los asuntos mundiales se ha convertido en su consigna cardinal, en medio de su discurso del "ascenso pacífico" del país y del "socialismo con características chinas". En cierta forma, el dinamismo militar actual repite la historia vivida por el Imperio del Centro dos siglos atrás cuando, en la decadencia de la dinastía Qing, las potencias imperialistas, con Inglaterra en la avanzada, se aprestaban a sacarlo de su enclaustramiento, con el solo propósito de multiplicar las adquisiciones de sus manufacturas milenarias y compensarlas con el ingreso masivo de opio, dándole un empuje colosal al mercado mundial por los británicos, al tráfico marítimo y a la banca dominados por los ingleses, así como para enriquecer las arcas reales. La diferencia sustancial con la situación presente se da en el hecho simple de que China no pasa por una fase de decadencia (García, 2009); sobre la base de su renacimiento productivo y político su proyección externa es, como nunca antes, asertiva y determinada.

Un país con tales características de jugador de primera línea en los asuntos globales impone por necesidad unas reglas para orga- 
nizar su área de influencia. Es inevitable, por tanto, que las instituciones asiáticas tengan un influjo directo de los intereses chinos. Acceder antes que Taiwán a la Organización Mundial del Comercio (omc) y ser el primero en participar en el esquema de integración comercial ASEAN +6 , revela un comportamiento propio de un país consciente de sus responsabilidades de superpotencia, lejos del modesto papel autoimpuesto en los tiempos de la Guerra Fría, cuando dejó a rusos y estadounidenses a merced de su desgaste mutuo. En su ascenso, los chinos insisten en la coexistencia pacífica, en medio de las solicitudes para que la comunidad mundial reconozca su rol político y militar central ${ }^{16}$. Junto al enriquecimiento de las organizaciones existentes, sus intereses son puestos al servicio de nuevos esquemas colaborativos, con el fin de no dejar vacío alguno a la injerencia regional de sus adversarios; de ahí el brío para concretar la cooperación centroasiática y el desenlace concertado de los planes atómicos norcoreanos (García, 2010).

Debido a estos desarrollos regionales apremiados por el desenvolvimiento de una potencia global como lo es China, para Japón será inevitable afrontar dilemas crecientes en torno a su defensa a partir de las obstrucciones en la alianza militar con Estados Unidos, si los recortes presupuestales en ambos países y el liderazgo económico del gran vecino animan la cooperación estratégica y política regional del este de Asia. Junto a ello se debe anotar el factor de la dependencia comercial, que da cuenta del $20 \%$ del intercambio externo japonés, con previsible aumento hasta llegar a duplicar los flujos con Estados Unidos, su socio más destacado en la posguerra ${ }^{17}$.

Las redefiniciones japonesas afrontan varias tendencias, en las que sobresalen la colaboración sino-rusa y la cooperación política y militar con el Sudeste Asiático ${ }^{18}$, ambas con repercusiones sobre el balance militar en Asia y el Pacífico heredado de la Guerra Fría. De esta forma, las opciones que sortearían los futuros dirigentes en Tokio estarían oscilando entre la acogida férrea del esquema de defensa actual y una asistencia con ciertas condiciones al liderazgo regional y global chino. Ambas posibilidades ya fueron ensayadas en los últimos ańos. El intento más cercano de trato distensionado con China se dio en 2009 cuando el público, cansado de la conducción doméstica e internacional del país en manos de la coalición derechista del PLD, depositó la confianza en las promesas del Partido Democrático de Japón, menos animoso respecto al establecimiento de acuerdos con la potencia comunista. En los tres ańos siguientes, varias reformas dirigidas a levantar la economía y mejorar las relaciones con

16 El White Paper de defensa sostiene que el país "se dotará de unas fuerzas armadas de defensa nacional poderosas acordes con la posición internacional de China y para responder a sus necesidades de seguridad y a sus intereses de desarrollo" (Xinhua, 2013).

17 El comercio con China presentó retroceso en 2012 a raíz de la tensión que entre ambos países produjo el incidente de las islas Senkaku/Diaoyutai (Jetro, 2013).

18 Que se viene consolidando por medios no tradicionales de cooperación política, con énfasis en la respuesta a los comunes problemas de seguridad (Arase, 2010). 
los vecinos motivaron gestos amigables con las autoridades de Beijing. En esa atmósfera de confianza, Yukio Hatoyama, Naoto Kan y Yoshihiko Noda evitaron, por ejemplo, los actos provocadores como el de la visita a las tumbas de los responsables de la guerra, en el santuario de Yasukuni, en Tokio. Para su desdicha, en el orden interno la confusión administrativa alrededor del terremoto de Fukushima desacreditó el mandato de Noda, en tanto que en el orden externo la contraposición con la República Popular sobre la soberanía de las Islas Senkaku/ Diaoyutai, alentada por la arenga ultranacionalistas de Shintaro Ishihara, justificó la retoma del Gobierno por el PLD en cabeza de Shinzo Abe, en diciembre de 2012.

Cabe esperar que una secuencia no muy larga de gobiernos conservadores insista en explotar el fervor nacionalista a favor de la recuperación productiva que contrarreste la rápida dependencia de las empresas japonesas de la mano de obra y los consumidores chinos, según la fórmula popularizada por Junichiro Koizumi después del año 2000, según la cual la alianza con Estados Unidos sería siempre "mejor opción" (Samuels, 2007). El cambio de orientación externa hacia la colaboración estrecha con los programas regionales y mundiales de la seguridad estadounidense también es previsible, de modo que las operaciones de Afganistán, Irak, Siria y otros frentes militares en Asia y África van a seguir contando con el soporte técnico, financiero y humano de las Fuerzas de Autodefensa. Más adelante, la asfixia presupuestal de Tokio y la necesidad de canalizar las oportunidades comerciales con China le abrirán las puertas del mando a una nueva coalición centrista no hostil al trato eco- nómico y político con la superpotencia vecina. Puesto que dentro de esos cambios abruptos, en comparación con las decisiones actuales, es factible encontrar a un Japón con menos resistencias frente al liderazgo chino, su política externa puede orientarse hacia la participación de la red de cooperación regional, a partir de los fundamentos históricos de la comunidad de valores confucianos, compartidos con un buen número de países de cercanos, como Corea, Taiwán y el Sudeste Asiático.

De esta manera, el sistema de cooperación asiático, sobre la base de la dependencia de las economías, abre la posibilidad de la concertación política y estratégica en desarrollos que se superponen a la experiencia positiva lograda con la ampliación de la ASEAN, y en la forma de capítulos inéditos, debido a que el celo por preservar los principios de no injerencia y respeto a los asuntos internos de los países detuvo hasta ahora la compaginación de áreas claves como la seguridad regional (Friedrichs, 2012). La probable colaboración con China no obliga a Japón a renunciar al acervo institucional y al acceso a los niveles directivos multilaterales. Esos logros contaron en el pasado con el respaldo estadounidense; sin embargo, retenerlos va a requerir el debido apoyo de Rusia y China, Estados con injerencia multilateral en crecimiento. Al mismo tiempo, los términos de la alianza militar con Estados Unidos tendrían que variar con el fin de mermar el personal estadounidense y el armamento desplegado en el Pacífico asiático, arsenal condenado por China y Rusia como provocador de la escalada armamentista en la zona. Ello justificaría un acuerdo para establecer medidas de confianza en términos de autonomía regional. 
Llegar a ese escenario colaborativo político y estratégico de Asia conlleva la premisa de un protagonismo chino efectivo, que tendría que resolver al menos tres condiciones: suficiente desarrollo económico como para elevar el presupuesto de defensa y de cooperación para el desarrollo del vecindario, el mejoramiento cualitativo del equipo de mando y la concertación con los demás gobiernos. Un rezago en el desempeño militar sería tan catastrófico para sus intereses nacionales como la imposición arbitraria de su plataforma de seguridad. El despliegue altanero, con pretensiones de policía regional y mundial, siguiendo el ejemplo de Estados Unidos, llevaría a Asia a luchas constantes y al mundo a una tensión impresionante, con diversos centros de conflicto en las zonas de presencia militar estadounidense, al igual que sobre las áreas de influencia de Rusia e India, entre otros países grandes.

Suele conjeturarse un futuro rompimiento entre las tendencias reformistas y conservadoras del Partido Comunista Chino, lo cual significa que la República Popular autoanularía su influencia en el orden global por venir (Ye, 2011). Esta hipótesis nos pone ante un anticipo apresurado ${ }^{19}$. Lo cierto es que hasta ahora la dirigencia china ha podido resolver sus diferencias internas de una forma poco traumática, despojándose de los elementos radicales, como el Bo Xilai, el exalcalde de Chongching, caído en desgracia y juzgado por corrupción. Desde marzo de 2013, el presidente Xi Jinping continuó los lineamientos de Hu Jintao, su predecesor, y como él, procura cierta firmeza en las reclamaciones territoriales sin llegar a tocar los límites de la agresión, lo que echaría por la borda las ambiciones chinas de liderazgo regional y global.

Esta actitud de autocontrol se ha visto clara en la cautela con que se viene manejando el asunto de las islas Spratley en el Mar del Sur de China y la tensión con Taiwán, isla con un gobierno propio que la dirigencia de Beijing no reconoce como tal y que, por tanto, le veta el ingreso a la onu. Sin duda, las medidas de fuerza para dirimir cualquiera de esas contiendas por parte de China tendrían severas consecuencias en cuanto a la competencia por lograr el favor de los Gobiernos en una ardua contienda con Washington. En efecto, la nueva propuesta de Barack Obama dirigida a reforzar su alianza tradicional militar con Australia, Japón y Corea, mediante el establecimiento de una asociación económica con ellos y otros países de la región (Malasia, Indonesia, Singapur y Vietnam) y algunos latinoamericanos (Chile, Perú y México), tiene el propósito de distanciarlos de la estrategia geopolítica de Beijing, encaminada a encajar los nuevos desarrollos de Asia y el Pacífico en el marco de sus intereses nacionales.

Así como la pugna entre China y Estados Unidos ocurre a pesar de la interdependencia económica, la rivalidad entre China y Japón reposa sobre la cruda realidad de dos países atados

19 A más largo plazo, China enfrentará la barrera formidable de la senilidad masiva. El fenómeno que ya soportan algunos países europeos, Corea y Japón, entre otros, se extenderá también a Rusia. Los trastornos económicos y sociales es probable que estén acompañados de recortes en sus programas de defensa (Sheen, 2013). 
por lazos que les son vitales. China le debe a las inversiones japonesas, que son las segundas después de las estadounidenses, buena parte de su auge industrial y, a su vez, el sostenimiento japonés como potencia económica estaría negado si tuviera que renunciar a las inversiones y las ventas de sus manufacturas en China. Este país no es solo el segundo mercado mundial sino que sobrepasó a Estados Unidos como el mayor consumidor de autos, un renglón rentable para los fabricantes japoneses.

En estas circunstancias es probable que Tokio atempere en forma progresiva su discurso antichino con el fin de no agravar la posición de las empresas japonesas en ese mercado, y al mismo tiempo el Gobierno chino busque ofrecerle alternativas a Japón con el fin de no forzarlo a incrementar la cooperación militar con Estados Unidos bajo la excusa de la amenaza externa. Por el contrario, medidas favorecedoras del ingreso de más industrias y productos japoneses remediarían la caída en los ingresos estatales japoneses y cimentaría la cooperación financiera que han explorado los países asiáticos para evitar los estragos de nuevas crisis como las soportadas desde el 2008 por parte de Europa y Estados Unidos.

Asimismo, la diplomacia china activa la concertación y la cooperación asiática en tres instancias: la ampliación de ASEAN a las economías grandes de Asia Oriental mediante el esquema ASEAN+6 y el fortalecimiento de la Organización de Cooperación de Shanghái, con Rusia y los países de Asia Central. Junto a ellos se vienen dando las cumbres del noreste asiático, reuniones periódicas de los jefes de Estado de China, Corea y Japón. Los diálogos complementarios dirigidos a facilitar los arreglos económicos y políticos por los canales bilaterales incluyen a India, Bangladesh y Birmania, en una posición destacada.

Habría un escenario alternativo de autonomía sobre la base de un Japón remilitarizado por cuenta propia. Ello implicaría la renuncia al Tratado de seguridad con Estados Unidos y la dotación de armamento original en un volumen y calidad tal como para disuadir cualquier proyección agresiva de los poderes militares vecinos, sean ellos Rusia, Sur Corea, China o Taiwán. Desde un punto de vista técnico y financiero parece una medida improbable, en cuanto supone producir armas balísticas y de detección y destrucción de misiles con tecnología endógena que las Fuerzas de Autodefensa no poseen, y cuya financiación iría en detrimento del nivel de vida popular. Al contrario, una colaboración con ciertas condiciones con China podría ser sugestiva para los políticos japoneses si la cooperación económica y social más estrecha llega a tener como compensaciones el arribo a la dirección estratégica mundial en el Consejo de Seguridad, la eliminación de la amenaza norcoreana y un acuerdo satisfactorio en las disputas territoriales con China, Corea y Rusia.

No se debe dejar de prever un escenario posterior definido por el agotamiento chino, ruso y de las principales economías asiáticas, como consecuencia de la factura demográfica. Su estancamiento contrastaría con el dinamismo productivo de otras potencias emergentes, India e Indonesia ante todo, que matizaría los arreglos intraasiáticos y la composición global del poder. Aún en estas condiciones, el esfuerzo que China y Rusia aplican al rescate de las reglas de juego multilaterales va a constituirse 
en un legado de sumo valor para resolver los conflictos entre los grandes poderes de forma pacífica. Allí también contará el compromiso que a lo largo de su existencia ha brindado Japón a la ONU.

\section{CONCLUSIÓN}

El marco de la geopolítica japonesa desde 1945, ha estado determinado por los intereses de Estados Unidos, país que tomó su control económico, político y militar una vez el emperador Hirohito aceptó la derrota. Entre ambos se tejió, desde entonces, una relación intensa extendida a los diversos campos de la producción industrial, las finanzas, los organismos multilaterales y la seguridad mutua. La colaboración japonesa al plan estratégico de Washington ha sufrido críticas que han dado lugar a ciertos cambios, como por ejemplo el traslado del cuerpo de marines a Guam; sin embargo, en sus términos básicos se preserva, aun en los momentos que el PLD ha estado fuera del poder. Las transformaciones en las relaciones de fuerzas en Asia del Pacífico, empero, vislumbran efectos sobre la alianza militar con Estados Unidos y las relaciones con los vecinos asiáticos. Respecto a estas últimas, un escenario de nexos más colaborativos alrededor de un programa de integración regional se perfila con alta probabilidad.

Por su capacidad económica y militar, China ejerce de una forma cada vez más visible la rectoría de los asuntos asiáticos. $\mathrm{Ha}$ llegado para ella el momento en que ve lejana la decisión de estar al margen de la contienda global como potencia media o como país no alineado, como fue su postura durante una buena parte de la Guerra Fría. Su traslado a la posición de balanceador del poder estadounidense y de sus aliados fue la consecuencia del vacío que generó el derrumbe soviético, y por esa misma dinámica defensiva y antihegemónica en el esquema de seguridad de Asia tiene influencia primordial el entendimiento con Rusia. A medida que Rusia y China convengan la política asiática, el interés por la resolución del conflicto regional de una forma más autónoma va a precisar la participación japonesa de manera mucho más decidida, con el efecto directo sobre la cooperación estratégica con Estados Unidos, pero con ganancias probables en la solución de sus disputas territoriales, el aplacamiento de los dolorosos recuerdos colectivos por sus agresiones expansionistas y la remoción de obstáculos para el reconocimiento mundial como miembro regular del Consejo de Seguridad.

Los entendimientos regionales tienen repercusiones inmediatas sobre los asuntos globales cuando se trata de actores con capacidades superiores. En la zona asiática encontramos tres de los cinco mayores gastos de defensa en manos de China, Rusia y Japón. Son, asimismo, potencias económicas cuyo intercambio ampliado refuerza un mercado de dinamismo notable. Como parte de la contraposición de fuerzas mundiales dentro de la cual se hallan envueltos esos países, la necesidad de concertación entre ellos a través de la cooperación regional es tan solicitada como su adhesión a los instrumentos multilaterales, lo cual significa que su voluntad de fortalecer al sistema de las Naciones Unidas será evidente. Al mismo tiempo, y debido a la réplica dialéctica de los 
movimientos entre los actores internacionales, el ordenamiento global más multilateralizado le planteará desafíos a los países asiáticos, forzando su concertación permanente. En este orden de ideas, la experiencia multilateral japonesa se convierte en un activo de una política exterior menos confrontadora en su entorno inmediato y más visible en el trabajo por alcanzar las metas convenidas a nivel global.

\section{REFERENCIAS}

Arase, D. (2010). Non-Traditional Security in ChinaASEAN Cooperation: The Institutionalization of Regional Security Cooperation and the Evolution of East Asian Regionalism. Asian Survey, $50(4), 808-833$.

Bilmes, L. (March de 2013). The Financial Legacy of Iraq and Afghanistan: How Wartime Spending Decisions Will Constrain Future National Security Budgets. Recuperado de: http://web.hks. harvard.edu/publications

Brzezinski, Z. (1998). The Grand Chessboard: American Primacy And Its Geostrategic Imperatives. New York: Basic Books.

Buzan, B. y Wrever, O. (2003). Regions and Powers. The Structure of International Security. Cambridge: Cambridge University Press.

Chanlett-Avery, E. Y. (3 de August de 2012). The U.S. Military Presence in Okinawa. Recuperado de: http://www.fas.org/sgp/crs/natsec/R42645.pdf

CIA. (25 de September de 2013). The World Factbook. Recuperado de: https://www.cia.gov/library/ publications/the-world-factbook/geos/ch.html

Ciorciari, J. D. (2011). Chian Mai Iniciative Multilateralization. Asian Survey, 52 (5), 926-952.
Eldridge, R. (2001). The Origins of the Bilateral Okinawa Problem: Okinawa in Postwar US-Japan. New York: Routledge.

Friedrichs, J. (2012). East Asian Regional Security. Asian Survey, 52 (4), 754-776.

Friedrichs, J. (2012). East Asian Regional Security. Asian Survey, 52 (4), 754-776.

Funabashi, Y. (1993). The Asianization of Asia. Foreign Affairs, 72 (5), 75-85.

García T. C. (2009). China. Su larga marcha hacia la globalización. Bogotá: Pontificia Universidad Javeriana/PROASIA Colombia.

García, P. (2001). El regreso del dragón. Geopolitica de Asia y el Pacífico. Bogotá: Universidad Externado de Colombia.

García, P. (2005). La relación China- ASEAN y la integración asiática. OASIS, 157-174.

García, P. (2010). Corea del Norte: vientos favorables para el cambio. Oasis, 14, 169-183.

He, K. Y. (2013). Prospects Theory and Foreign Policy in the Asia Pacific. Rational leaders and Risky Behavior. New York: Routledge.

Hughes, C. (2009). "Super-Sizing" the DPRK Threat: Japan's Evolving Military Posture and North Korea. Asian Survey, 49 (2), 291-311.

Jetro (19 de February de 2013). Jetro survey: Analysis of Japan-China Trade in 2013 and Outlook for 2013. Recuperado de: http://www.jetro.go.jp/en/ news/releases/20130219452-news

Johnson, C. (1982). MITI and the Japanese Miracle: The Growth of Industrial Policy, 1925-1975. Stanford: Stanford University Press.

Kahn, H. (1970). The Emerging Japanese Superstate: challenge and response. New Jersey: Prentice Hall.

Kihl, Y. W. (1986). Asian-Pacific Security. Emerging Challenges and Responses. Boulder: Lynne Rienner Publishers. 
Mearsheimer, J. (2001). The Tragedy of Great Power Politics. New York: W. W. Norton \& Co.

Mearsheimer, J. J. (2006). China's Unpeaceful Rise. Current History (105), 160-162.

Nakamura, T. (1988). El Desarrollo Económico del Japón Moderno. México D. F. : El Colegio de México.

OECD. (29 de May de 2013). Government Debt. Economics: Key Tables from OECD (21). Recuperado de: http://www.oecd-ilibrary.org/economics/ government-debt_gov-debt-table-en

Packard, G. (2010). The United Sates- Japan Security Treaty at 50. Foreign Affairs, 89 (2), 92-103.

Rhyu, S. Y. (2006). Changing Dynamics in Korea-Japan Economic Relations: Policy Ideas and Development. Asian Survey, 46 (2), 195-214.

Salem Press. (April de 2009). Japanese Trade with the United States. Recuperado de: http://salempress. com/store/samples/american_business/american_business_japanese.htm

Samuels, R. J. (2007). Securing Japan. Tokyo's Grand Strategy and the Future of East Asia. Ithaca: Cornell University Press.

Sheen, S. (2013). Northeast Asia's Aging Population and Regional Security: Demographic Peace? Asian Survey, 53 (2), 292-318.

Stengel, F. (January de 2008). The Reluctant Peacekeeper: Japan's. Recuperado de: http://www. giga-hamburg.de/dl/download.php?d=/content/publikationen/archiv/ja_aktuell/jaa_0801_ fokus_stengel.pdf

Takenaka, K. (22 de February de 2013). On key U.S. visit, Abe vows to bring back a strong Japan. Recuperado de: http://www.reuters.com/article/2013/02/22/us-usa-japan-abe-idUSBRE91L12P20130222
Times, T. S. (8 de October de 2013). Invest in Siberia' - Putin call to Asia-Pacific business partners. Recuperado de: http://siberiantimes.com/business/ investment/news/invest-in-siberia-putin-call-toasia-pacific-business-partners/

Trading Economics (June de 2013). Japan Balance of Trade. Recuperado de: http://www.tradingeconomics.com/japan/balance-of-trade

Vogel, E. (1980). Japan as Number One: Lessons for America. Cambridge: Harvard University Press.

Wallerstein, I. (1974). The Rise and Future Demise of the World Capitalist System: Concepts for Comparative Analysis. Comparative Studies in Society and History, 16 (4), 387-415.

Wishnick, E. (February de 2009). Russia, China, and the United States in Central Asia: Prospects for Great Power Competition and Cooperation in the Shadow of the Georgian Crisis. Recuperado de: http://www.strategicstudiesinstitute.army.mil/ pubs/summary.cfm?q=907

Woronoff, J. (1992). Asia's 'Miracle' Economies. New York: M. E. Sharpe.

Wortzel, L. M. (2013). The Dragon Extends its Reach: Chinese Military Power Goes Global. Dulles, VA: Potomac Books.

Xinhua (16 de April de 2013). China issues national defense white paper. Recuperado de: http:// news.xinhuanet.com/english/china/201304/16/c_132312449.htm

Ye, Z. (2011). Inside China's Grand Strategy. The Perspective from the People's Republic. Lexington: The University Press of Kentucky.

Yoda, T. (2006). Japan's Host Nation Support Program for the U.S.-Japan Security Alliance: Past and Prospects. Asian Survey, 46 (6), 937-961. 